\title{
Silicon Photonics for Optical Access Networks
}

\author{
Ryohei Urata, Hong Liu, Cedric Lam, Pedram Dashti, and Chris Johnson \\ Google Inc., 1600 Amphitheatre Parkway, Mountain View, CA 94043, USA \\ ryohei@google.com
}

\begin{abstract}
We highlight promising developments and directions in silicon photonics for realizing cost effective WDM-PON: photonic integration for integrated WDM transceivers at the OLT and widely tunable laser technologies for achieving a high performance, colorless ONU. OCIS codes: (060.2330) Fiber optics communications; (060.4250) Networks; (140.3600) Lasers, tunable
\end{abstract}

\section{Introduction}

In the past several years, there has been tremendous growth of web-based applications, cloud computing, and content streaming. This trend requires a commensurate increase in end-user/access bandwidth for delivering services in a quality manner and enabling new, more bandwidth-hungry applications in the future.

Build outs of access network infrastructure are extremely capital intensive, thus requiring a future-proof infrastructure which can ensure bandwidth scalability for an extended period of time. With this consideration, fiber is the only future-proof broadband access medium and fiber-to-the-home (FTTH) services have been successfully rolled out in various deployments throughout the world.

Up to this point, FTTH deployments have predominantly utilized a time division multiplexed (TDM)-passive optical network (PON) scheme due to simplicity of fiber plant implementation and the ability of technologies to easily support the desired data rates at the time of adoption. Illustrated in Fig. 1a), a shared optical line terminal $(\mathrm{OLT})$ transceiver at the central office (CO) broadcasts downstream to individual optical network units (ONUs), where the correct packets are selected out as part of the PON protocol. In the reverse, upstream direction, transmission occurs in a burst mode fashion to allow time sharing of the single communication channel. However, as the need for bandwidth increases, it is clear that this traditional PON architecture will not be able to scale, as all transceivers must work at the aggregate bit rate of the entire system. In addition, the unique protocol required for TDM-PON to function does not allow end-to-end Ethernet protocol transparency, with a cumbersome conversion needed from TDM-PON protocol to Ethernet (and back) at all end points.

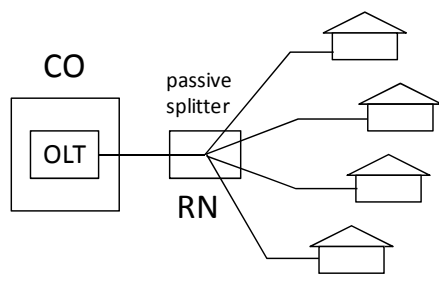

(a) TDM-PON

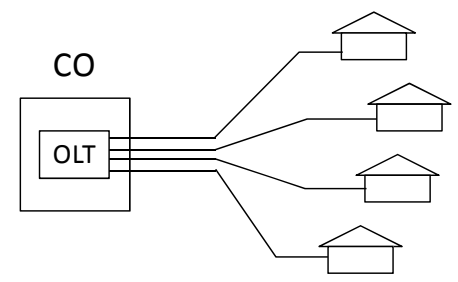

(b) point-to-point

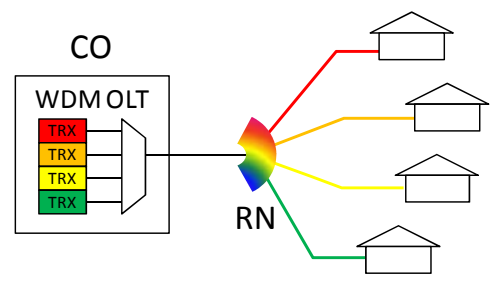

(c) WDM-PON

Fig. 1: Simple illustrations of a) TDM-PON, b) point-to-point, and c) WDM-PON.

The second approach is to employ a point-to-point scheme, in which an individual fiber is connected from the OLT to each ONU, with a dedicated fiber for each user (Fig. 1b)). From a performance perspective, point-to-point is superior in its ability to scale both bandwidth and transmission distance. However, these advantages are offset by the significant cost increase in most cases, resulting from implementing a large number of lines for the fiber infrastructure. The number of fibers and terminations also complicates maintenance and management.

WDM-PON, using wavelength division multiplexing (WDM), has long been touted as the key to delivering symmetric and sustained bandwidth at or beyond $1 \mathrm{~Gb} / \mathrm{s}$ to each user. Higher security can also be an added benefit. Over the years, a variety of implementations and corresponding device technologies have been investigated as a result [1]. In this paper, we discuss the potential role of silicon photonics for realizing key device technologies which enable a high performance, robust, and cost effective WDM-PON. In particular, we highlight promising developments and directions in silicon photonics for integrated WDM transceivers at the OLT and widely tunable laser technologies for achieving a high performance, colorless ONU. 


\section{WDM-PON System}

Fig. 1c) illustrates the WDM PON system. The CO contains multiple transceivers at different wavelengths with each output wavelength creating a dedicated path or channel for a particular user by passing through a wavelength selective/dependent element at the remote node $(\mathrm{RN})$. Wavelength selection can also be achieved by filtering at the user (broadcast and select). The upstream connection similarly utilizes a dedicated wavelength channel. With this architecture, OLT and ONU transceivers need only operate at the data rate directly seen by the user, the Ethernet protocol is preserved from end-to-end, and the fiber infrastructure is equivalent to TDM-PON. WDM-PON thus combines the bandwidth scalability of point-to-point and the fiber consolidation of TDM-PON. However, these advantages are accompanied by challenges in realizing the transceivers at the end points, as well as the remote node.

An arrayed waveguide grating (AWG) technology can realize the remote node, but passive operation is needed to reduce cost, power consumption, and probability of failure. There have been significant advancements in athermal arrayed waveguide grating (AWG) technology [2], paving the way for a compact, monolithic remote node which exhibits good performance over temperature hardened conditions $\left(-40\right.$ to $\left.+85^{\circ} \mathrm{C}\right)$. High delta waveguides allow tight bends, leading to a compact cartridge size comparable to that for a splitter.

\section{A. WDM transceiver OLT}

At the OLT, adopting individual transceiver units for each WDM channel increases size, cost, and power. Thus, for the $\mathrm{CO}$, the integration of the transmit, receive, demux, and mux components into an integrated WDM transceiver is critical. This can be achieved through either monolithic integration, hybrid integration, and/or novel packaging techniques [3]. Monolithic integration has been a long running theme and goal for the photonics community, with steady progress leading to recent wide-scale deployment of large-scale, InP-based photonic integrated circuit (PIC)based solutions [4]. However, there are still issues with chip yield as device counts increase. The prohibitive cost of maintaining a clean room capable of large-scale integration also limits the number of players and bottlenecks both innovation and cost. In addition, for extending to higher data rates beyond $1 \mathrm{Gbps}$, it is likely integration of external modulators will be needed (as opposed to simple direct modulation), further complicating the fabrication process and reducing yield. There have been suggestions of establishing a foundry model analogous in approach to the silicon IC industry [5]. Still, it is not clear that the volume of PICs would allow this model to be successful.

The alternative approach is that of hybrid integration, where most commonly, active sections are realized in III$\mathrm{V}$ materials and passives in silica/silicon, taking and combining the strengths of both material systems [6,7]. Although improvement in critical packaging and manufacturing processes such as passive alignment techniques have improved significantly over the years, the ultimate in performance and cost is still not realized.

On the other hand, silicon photonics [8] leverages a mature, highly repeatable process which potentially allows: 1) photonic and electronic integrated circuits with large-scale integration for superior functionality, 2) improved performance and power consumption as a result of intimate integration of photonic and electronic devices, 3 ) lower integration/packaging cost, 4) cost sharing with traditional all-electronic silicon ICs assuming photonics can be supported within a standard (or near standard) CMOS process.

A number of challenges remain, realization of the laser in particular. Approaches include bonding techniques [9] and alignment of individual or arrays of lasers to a silicon bench [10,11], and a number of WDM transceivers have been proposed or demonstrated based on these various approaches [11-15]. In addition, realization of silicon photonic external modulators allows a graceful upgrade path to higher data rates $(\geq 10 \mathrm{Gbps})$.

It is important to note that for application in access networks, depending on the implementation of the RN, wavelength accuracy may be relaxed in comparison to traditional DWDM systems. This would allow relaxed performance specifications (applicable to all integration approaches) for enhancing manufacturability and elimination of the wavelength locker to further simplify the transceiver.

\section{B. Colorless ONU}

A colorless ONU supporting all wavelength channels is needed in order to ease operation and maintenance and reduce cost through volume production of one component. Although a variety of creative schemes and devices have been investigated [16, 17], from a performance standpoint, the availability of an ONU with a wavelength tunable laser as the transmitter will provide superior signal quality, reach, and stability, with the wavelength of each ONU tuned to the appropriate upstream channel. The underlying issue has been the cost of the widely tunable lasers being unable to reach the cost points required for adoption in access networks.

A majority of the tunable lasers currently deployed are distributed Bragg reflector (DBR)-type [18-20]. Similar to WDM transceivers, the idea was that monolithic integration of the gain, phase, and tuning elements could be 
leveraged to reduce size, cost, and power. However, the complicated fabrication which requires electron beam or high resolution lithography for defining the gratings and in particular, the need to bury the gratings with a regrowth step, can impact final device yield, although significant progress has been made over the years [20].

An alternative approach is to simplify the required III-V semiconductor block to the limit, with only the gain block being fabricated in InP-based material and the ring resonator tuning elements realized in a silicon photonics platform [21,22]. Heating of the rings induces a thermo-optic effect which shifts the resonant wavelengths of the rings and hence, the lasing wavelength. The silicon microrings exhibit high $\mathrm{Q}$, allowing reduced tuning power. The independent optimization and high yield of the various components is at the expense of packaging complexity and the need to achieve good optical alignment and reduce reflections at the III-V/silicon interface. The latter two are resolved with a SiON/Si waveguide and spot-size converter in [21]. The coupling issue can also be resolved using a broadband grating coupler with large alignment tolerance [22].

An additional advantage of the silicon photonics approaches is wavelength stability, as the tuning elements are used in a passive manner, as opposed to most monolithic approaches which require carrier injection into the waveguide itself and gradually degrade. This increased wavelength stability, along with the reduced spec in wavelength accuracy noted earlier, increases manufacturability and eliminates the wavelength locker. One key challenge for all tunables is reducing the required testing time to increase manufacturing throughput [20].

\section{Conclusion}

The advantages of silicon photonics are reliable integration and functionality at low cost, with the prerequisite of high volume. The access domain promises the highest volumes of any telecom space and WDM PON is the most promising bandwidth-scalable solution, which requires integration and functionality to achieve key technologies at the OLT and ONU. With this match in advantages and requirements, access networks may be the right driver for enabling silicon photonics into the mainstream.

\section{References}

[1] J. Kani, "Enabling technologies for future scalable and flexible WDM-PON and WDM/TDM-PON systems," IEEE J. Sel. Topics Quantum Electron., vol. 16, no. 5, pp. 1290-1297, Sept./Oct. 2010.

[2] S. Kamei, "Recent progress on athermal AWG wavelength multiplexer," OFC/NFOEC 2009, OWO1.

[3] H. Liu, C.F. Lam, and C. Johnson, "Scaling optical interconnects in datacenter networks," IEEE Symposium on High Performance Interconnects 2010.

[4] R. Nagarajan, et al., "Large-scale photonic integrated circuits," IEEE J. Sel. Topics Quantum Electron., vol. 11, no. 1, pp. 50-65, 2005.

[5] M. Smit, et al., "Generic foundry model for InP-based photonics," IET Optoelectronics, vol. 5, iss. 5, pp. 187-194, 2011.

[6] G. Maxwell, et al., "Hybrid integration of monolithic semiconductor optical amplifier arrays using passive assembly," IEEE Electronic Components and Technology Conference 2005, vol. 2, pp. 1349-1352.

[7] T. Schrans, et al., "100Gb/s 10km link performance of 10x10Gb/s hybrid approach with integrated WDM array of DFB lasers," OFC 2009, NThA4.

[8] Y.A. Vlasov, "Silicon CMOS-integrated nano-photonics for computer and data communications beyond 100G," IEEE Communications Magazine, S67-S72, Feb. 2012.

[9] A. Alduino et al., "Demonstration of a high speed 4-channel integrated silicon photonics WDM link with hybrid silicon lasers," Integrated Photonics Research (IPR) 2010, PDIWI5.

[10] M. Asghari, "Silicon photonics: A low cost integration platform for datacom and telecom applications," OFC 2008, NThA4.

[11] S. Assefa, W.M.J. Green, A. Rylyakov, C. Schow, F. Horst, and Y.A. Vlasov, "CMOS integrated nanophotonics - enabling technology for exascale computing systems," OFC 2011, OMM6.

[12] B. Koch, et al., "A 4x12.5Gb/s CWDM Si photonics link using integrated hybrid silicon lasers," CLEO 2011, CThP5.

[13] P. De Dobbelaere, et al., "Demonstration of first WDM CMOS photonics transceiver with monolithically integrated photo-detectors," ECOC 2008, Tu.3.C.1.

[14] A. Narasimha, B. Analui, Y. Liang, T.J. Sleboda, and C. Gunn, “A fully integrated 4x10Gb/s DWDM optoelectronic transceiver in a standard 0.13um CMOS SOI," ISSCC 2007, 2.1.

[15] D. Feng et al., "Terabit/s single chip WDM receiver on the SOI platform," Group IV Photonics 2011, FA2.

[16] F. Payoux, P. Chanclou, and R. Brenot, "WDM PON with a single SLED seeding colorless RSOA-based OLT and ONUs," ECOC 2006, Tu4.5.1.

[17] S. Park, C. Lee, K. Jeong, H. Park, J. Ahn, and K. Song, "Fiber-to-the-home services based on wavelength-division-multiplexing passive optical network,” IEEE J. Lightw. Technol., vol. 22, no. 11, pp. 2582-2591, Nov. 2004.

[18] V. Jayaraman, Z.-M. Chuang, and L.A. Coldren, "Theory, design, and performance of extended tuning range semiconductor lasers with sampled gratings," IEEE J. Quantum Electron., vol. 29, no. 6, pp. 1824-1834, Jun. 1993.

[19] H. Ishii, Y. Tohmori, Y. Yoshikuni. T. Tamamura, and Y. Kondo, "Multiple-phase shift super structure grating DBR lasers for broad wavelength tuning," IEEE Photon. Technol. Lett., vol. 5, no. 6, pp. 613-615, Jun. 1993.

[20] A. C. Carter, "Evolution of optical component technologies for access and metro networks," ECOC 2009, Paper 9.5.1.

[21] N. Fujioka, T. Chu, and M. Ishizaka, "Compact and low power consumption hybrid integrated wavelength tunable laser module using silicon waveguide resonators," IEEE J. Lightw. Technol., vol. 28, no. 21, pp. 3115-3120, Nov. 2010.

[22] G. Yoffe, T. Nguyen, J. Heanue, and B. Pezeshki, "Efficient compact tunable laser for access networks using silicon ring resonators," OFC 2012, OW1G.4. 